\title{
The Müller-Lyer contrast illusion: A computational approach
}

\author{
GORDON M. REDDING, GEORGIA D. WINSON, and RICHARD O. TEMPLE \\ Illinois State University, Normal, Illinois
}

\begin{abstract}
When a temporal delay is interposed between the contextual elements (wings) and the focal element (central axis) of the Müller-Lyer figures, the usual assimilation illusion changes to an illusion of contrast; that is, judged axis length is contrasted away from rather than assimilated toward the context provided by parallel extents between wings. Presentation time for the preceding contextual wings on the order of $1 \mathrm{sec}$ or more was needed to produce contrast effects in judgments of the following focal axis (Experiment 3) and, given sufficient presentation time, these contrast effects were largely unaffected by the length of the temporal delay between contextual and focal elements, appearing equally strong for delays between 0 and $2 \sec$ (Experiments 1 and 2). These results are consistent with a representational basis for these contrast effects that is high-level and long-lived. The Müller-Lyer contrast illusion may reflect the inadvertent error arising from basing judgments about particular objects on information about attribute differences among objects. Such judgmental errors may be the natural consequence of constrained computations that make explicit information required for certain common tasks, but at the expense of obscuring information required for less common tasks.
\end{abstract}

When a temporal delay is interposed between the contextual elements ("wings" or "fins") and the focal element (central axis or "shaft") of Müller-Lyer figures, the usual assimilation illusion changes to an illusion of contrast; that is, judged axis length is contrasted away from rather than assimilated toward the context provided by parallel extents between wings (Jordan \& Uhlarik, 1986; Pollack, 1964) ${ }^{1}$ In Figure 1, the assimilation and contrast effects obtained by Jordan and Uhlarik have been reproduced. Similar results have also been found when contextual elements were presented spatially separated from (i.e., above) focal elements, requiring eye movement for foveation (Jordan \& Uhlarik, 1986, Experiment 2), and when context was defined by a set of parallel lines rather than the Müller-Lyer wings (Jordan \& Schiano, 1986; Jordan \& Uhlarik, 1985).

These results support the general proposal (Coren \& Girgus, 1978; see also Coren \& Girgus, 1980, Girgus \& Coren, 1982) that assimilation effects arise from perceptual "pooling"' of simultaneously occurring stimulus elements (cf. Pressey, 1970, 1972, 1974), whereas contrast effects arise from the encoding of differences due to the need to "store"' successively occurring stimulus elements. Jordan and colleagues (Jordan \& English, 1989; Jordan \& Haleblian, 1988; Jordan \& Schiano, 1986; Jordan \& Uhlarik, 1985, 1986) have more clearly identified simul-

A preliminary report of Experiments 1 and 2 was made at the 64th annual meeting of the Midwestern Psychological Association, Chicago, IL. The authors with to thank the following participants in an informal "illusions discussion group" for their critique of the present data: Christopher Ege, Erik Hawley, Michele Johnson, Amy Shelton, and Richard Temple. Address correspondence to G. M. Redding, Department of Psychology, Illinois State University, Normal, IL 61761-6901. taneous and successive conditions. Stimulus elements are simultaneous when they occur within the focus of spatial attention (cf. Pressey \& DiLollo, 1978) and successive when they occur in different shifts of focal attention, with or without eye movement (e.g., Posner, 1980). The elaborated "pool-and-store" model clearly specifies differential conditions for the two kinds of distortions, but it is less clear about what processes underlie the illusions. What exactly is "pooling" and "storage"? Perhaps more importantly, why should the visual system include processes that misrepresent the world? Some answers to these questions are suggested by consideration of what kinds of information are made explicit in early vision and what kinds of behavior are supported by the information-that is, a "computational" approach.

Reportable perceptual experience can be viewed as the product of computational processes that, beginning with the retinal intensity array, construct incremental representations (informational structures) of increasing levels of abstraction, arriving at high-level representations that correspond in important respects to the distal stimulus (e.g., Marr, 1982; Marr \& Poggio, 1977). Necessary to such computations is the application of organizing principles that reflect implicit knowledge ("assumptions") of physical regularities (e.g., of image formation and projective geometry) to constrain interpretation of the proximal stimulus (see also Biederman, 1987; cf. Ittelson \& Kilpatrick, 1951). Evolutionary pressures can be assumed to have favored constrained computations that extract information for perceptual-motor tasks having a high survival value. However, the consequence of such natural selection may be that the requisite information for some other tasks may not be explicitly available, especially for 


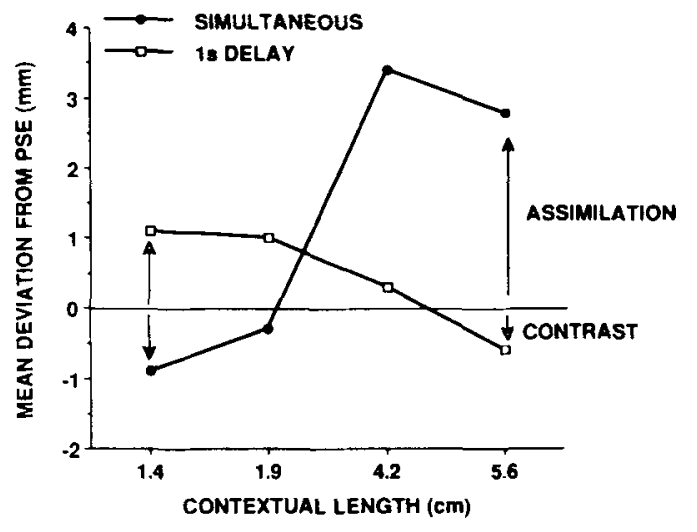

Figure 1. Data from Jordan and Uhlarik (1986, Experiment 1). Mean deviation from point of subjective equality (PSE) for judgments of $2.8-\mathrm{cm}\left(2.0^{\circ}\right)$ focal length (central axis) as a function of inward wings produced by 1.4 and $1.9-\mathrm{cm}$ contextual lengths $\left(1.0^{\circ}\right.$ and $1.3^{\circ}$ ) and outward wings produced by 4.2 - and $5.6-\mathrm{cm}$ contextual lengths $\left(3.1^{\circ}\right.$ and $\left.4.1^{\circ}\right)$ for conditions in which contextual elements preceded the focal line by a dark interval (1-sec delay) or contextual and focal elements appeared together (simultaneous). Directions for assimilation and contrast effects are indicated. Note that contextual length is defined as inter -wing-tip distance (See Figure 3 and corresponding text).

those that are experimentally defined. In such cases, the visual system does the best that it can with the available machinery. "What we call illusions arise when the visual system is asked to perform some task to which it is illadapted"' (Morgan, Hole, \& Glennerster, 1990, p. 1800).

For example, Morgan et al. (1990; see also Morgan \& Glennerster, 1991) argue that the Müller-Lyer assimilation illusion arises from positional misjudgments of shaft terminations induced by the wings (cf. Chiang, 1968; Coren, 1970, 1986; Holding, 1970; Stuart, Day, \& Dickinson, 1984) and, more importantly, that such misjudgments reflect the natural bias of the visual system toward representing the position of multipart stimuli with a single average value, or "centroid." The distance between encoded shaft terminations (i.e., centroids) is greater for outwardwings stimuli than for inward-wings stimuli. An important advance over similar proposals (e.g., Pressey $\&$ Martin, 1990 ) is Morgan et al.'s suggestion of the computational constraints underlying positional misjudgment. Abstracting the average position of an object well serves the needs of manipulation and locomotion, but such an adaptation may make it difficult to extract the position of parts of an object independently of immediately surrounding parts, even though the parts may be visually resolvable.

We suggest that position averaging and consequential assimilation effects in judgments of positional separation may be a specific instance of a more general principle describing the manner in which representations of discrete objects are formed. Treisman (1985; Treisman \& Gelade, 1980 ) argues that early visual processing extracts spatially coded information about the several attributes of an object (e.g., shape, size, color, orientation), which must then be bound together within the focus of attention to create a unitary object representation. Implicit in such unitizing computation is the general constraint (assumption) that discrete objects (usually) have single values of the several attributes. Even when an object contains resolvable parts having different values of an attribute, it may be represented as a whole by a single average value. Thus, attribute averaging may be a general characteristic of the constrained computations that create unitized representations of discrete objects present in the visual field. Such unitized representation would be largely veridical for actions directed toward the whole object, but may be nonveridical for actions directed at different parts of the object.

A similarly general computational constraint may underlie the Müller-Lyer contrast illusion. Ullman (1984; see also Minsky \& Papert, 1969) has argued that the comparison computations underlying the perception of spatial relations (e.g., inside/outside, above/below, larger than/ smaller than) require spatially sequential processing at higher levels of the visual system. Unlike lower level representational processes, which can be largely stimulus driven, relational judgments are more task specific and therefore require the strategic flexibility of sequential processing. We suggest that relational comparisons are computed after object representation is complete. Implicit in such comparison computation is the general constraint (assumption) that different objects (are likely to) have different attribute values. Even when objects are not perceptibly different in absolute terms, direct comparison may reveal differences in attribute values. There may be inhibitory connections between object-level representations that operate to enhance differences in attribute values, under the assumption (constraint) that a relative difference in attribute value (likely) represents an absolute difference. Thus, attribute differencing may be a general characteristic of the constrained computations that extract spatial relations among representations of discrete objects present in the visual field. Such spatial comparison would be largely veridical for actions directed toward the collection of objects in the visual field, but may be nonveridical for actions directed to individual objects.

More specifically, for the Müller-Lyer stimuli, when contextual wings and the focal axis are processed in different shifts of focal attention, as when they are spatially separated or equivalently separated in time, they may be represented as distinct objects. Attribute averaging in the formation of object representations would tend to misrepresent linear extent between the contextual wings, but not for the focal axis. Subsequently, the resultant difference in linear extent between representations of the two objects would tend to be exaggerated in higher level computation of the larger than/smaller than relation set by the size judgment task (i.e., contrast effects). The preceding outwardwings context produces smaller judgments of focal extent, whereas the inward-wings context produces larger judgments.

In summary, we suggest that assimilation effects arise from low-level averaging processes that create a unitized 
object representation of stimulus elements within the focus of attention, whereas contrast effects arise from highlevel differencing processes that preserve the uniqueness of representations acquired by successive changes in the focus of attention. In Figure 2, the different kinds of processes and resultant differential effects are schematically illustrated. Consistent with the view that the usual MüllerLyer assimilation illusion is an effect of lower levels of visual processing is the observation that this illusion is affected by variables such as lightness contrast and tachistoscopic viewing time (e.g., Ebert \& Pollack, 1972, 1973; Erlebacher \& Sekular, 1974). Moreover, the fact that the assimilation illusion declines in magnitude with increasing age, whereas the contrast illusion increases, further suggests that the two illusions arise from different levels of processing (e.g., Piaget, 1969; Pollack, 1964). Several new predictions are possible from the view that the Müller-Lyer contrast illusion arises from high-level computation of spatial relations.

First, contrast effects should be small in magnitude, relative to the usual assimilation effects. The nature of constraints is to reduce ambiguity. In computing spatial relations among object representations, the general constraint operates toward assuming differences when there is uncertainty. If two objects are obviously different, there should be less exaggeration of the difference. On the other hand, assimilation effects may be limited only by the range of contextual extents within the focus of attention. A corollary is that contrast effects should be largest where assimilation effects are smallest. Small assimilation effects will produce small differences in linear extent between the contextual wings configuration and focal axis with consequent exaggeration in computing the linear extent relationship between the two representations. On the other hand, large assimilation effects will produce large differences between context and focal representations with less exaggeration of the difference. The data from Jordan and Uhlarik (1986) displayed in Figure 1 are consistent with these predictions
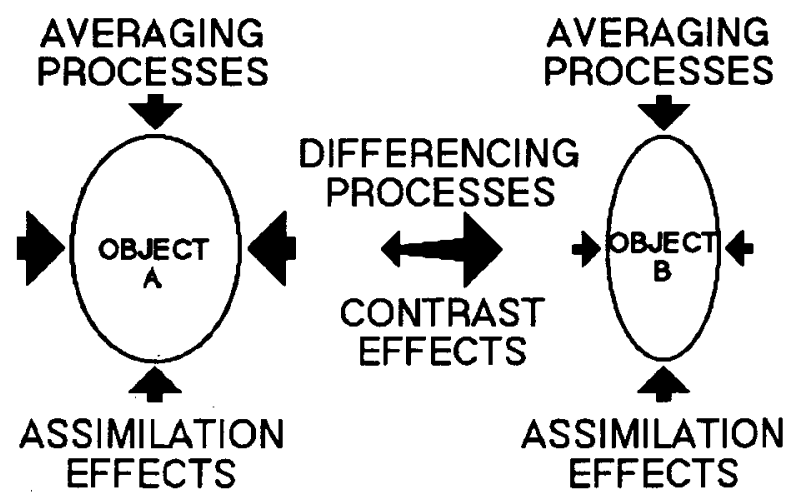

Figure 2. Hypothetical representational processes that might operate on successively attended objects to produce assimilation and contrast effects. Averaging processes may produce underestimation of large extents and overestimation of small extents in the unitized representation. Differencing processes may reduce representations of small extent relative to representations having a larger extent. in showing overall smaller contrast than assimilation, and greatest contrast where the least amount of assimilation occurred; namely, for inward-wings configurations. ${ }^{2}$

Second, if contrast effects are based on comparisons among high-level (object) representations, they should be immune to any low-level integration of information from successively viewed stimuli, provided that sufficient time is allowed for development of the base representations. More specifically, the effective lifetime of such representations should be in terms of high-level vision (e.g., seconds) rather than low-level vision (e.g., milliseconds) and the contrast illusion should appear, even with long delays between contextual and focal elements. To investigate this hypothesis in the present Experiments 1 and 2, we parametrically manipulated the dark delay between contextual and focal elements, thereby varying the opportunity for low-level integrative processes, while holding presentation time of the separate elements constant at a value known to be sufficient for contrast effects and presumably for development of high-level representations.

Third, if insufficient time is allowed for development of high-level representations, contrast effects should not appear. More specifically, development of high-level object representations should require relatively long stimulus presentation times, on the order of substantial portions of $1 \mathrm{sec}$ or greater. If stimulus presentation time is insufficient, high-level representations will not be available for the comparison computations presumed to be responsible for contrast effects. In Experiment 3, we investigated this hypothesis by manipulating the duration of the contextual wings while employing a pattern mask between contextual and focal elements to limit processing of the contextual elements to presentation time.

\section{EXPERIMENTS 1 AND 2}

In the first two experiments, we investigated contrast effects as a function of duration of the dark delay between contextual and focal elements. The principal difference between Experiments 1 and 2 was the range of this temporal delay.

\section{Method}

The method employed in the first two experiments was based on Jordan and Uhlarik's (1986) Experiment 1, but with a manipulated dark interval between contextual and focal stimuli instead of a constant interstimulus interval. Also like Jordan and Uhlarik, a condition with contextual and focal stimuli presented simultaneously was included for comparison.

Subjects. The 80 subjects in each experiment were undergraduate students enrolled in psychology courses who volunteered to participate in return for course credit. By self-report, all the subjects had either normal or corrected-to-normal vision.

Design. In each experiment, four random groups (20 subjects each) experienced the different conditions, which are illustrated in Figure 3 . The simultaneous group experienced contextual (wings) and focal (central axis) elements simultaneously, as in the usual Müller-Lyer configuration. For the 0 -sec-delay group, focal elements were presented separately, but immediately followed contextual elements. For two other dark-delay groups, contextual and focal stimuli were separated by different intervals, during which 
SIMULTANEOUS CONDITION

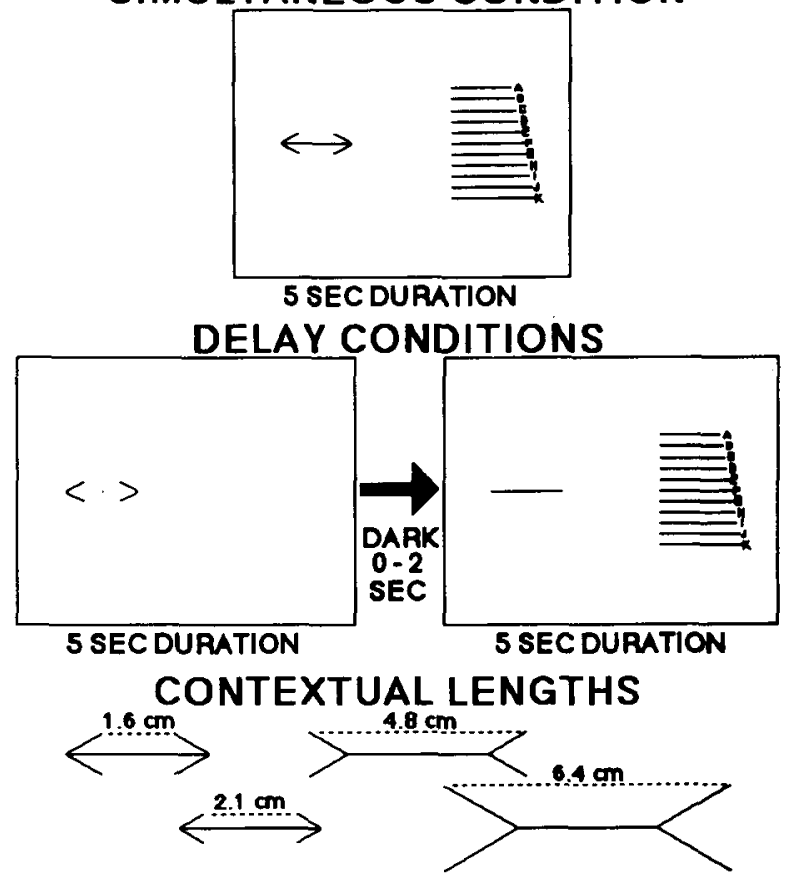

Figure 3. Illustration of experimental conditions and levels of contextual length for Experiments 1 and 2. In the simultaneous condition, contextual wings were presented simultaneously with the focal central axis and comparison series for 5 sec. In the delay conditions, the 5-sec presentation of focal central axis and comparison series immediately followed the 5 -sec presentation of contextual wings (0-sec delay) or a dark interval was interposed between the two stimulus fields. Contextual lengths are illustrated as inter-wing-tip distance.

time the visual field was dark. Dark delays of .5 and $1 \mathrm{sec}$ were used in Experiment 1; the range of delay was increased to 1 and $2 \mathrm{sec}$ in Experiment 2. Duration of simultaneous presentations was $5 \mathrm{sec}$. In the delay conditions, contextual and focal elements were each presented for $5 \mathrm{sec}$, and a small fixation dot ( $1 \mathrm{~mm}$ diameter) was centered between the contextual elements.

All stimulus events occurred in an Iconix four-field tachistoscope with a viewing distance of $90 \mathrm{~cm}$. The following stimulus parameters were selected to closely approximate visual angle equivalents of the values used by Jordan and Uhlarik (1986). Focal length was constant at $3.2 \mathrm{~cm}\left(2.0^{\circ}\right)$ and four contextual lengths were defined for $60^{\circ}$ angles between wings as the distance between wing tips$1.6,2.1,4.8$, or $6.4 \mathrm{~cm}\left(1.0^{\circ}, 1.3^{\circ}, 3.1^{\circ}\right.$, or $\left.4.1^{\circ}\right)$. As can be seen in Figure 3, the two shorter contextual lengths produced the usual inward-wings stimuli and the two longer contextual lengths produced the outward-wings stimuli. These four experimental stimuli together with two filler stimuli were presented in four randomized blocks of trials; that is, each of the six stimuli was presented once in each of the four blocks for a total of 24 experimental trials. The filler stimuli were 2.8 - and $3.6-\mathrm{cm}\left(1.8^{\circ}\right.$ and $\left.2.3^{\circ}\right)$ lines without wings. These fillers were intended to encourage the subjects to use the full range of comparison stimuli in their judgments. Line thickness was constant at $.6 \mathrm{~mm}\left(.04^{\circ}\right)$ for all stimuli.

Procedure. For practice, the subjects judged six lines without wings presented in a random order, including two repetitions of the experimental focal length that were used to provide a baseline control measure; that is, $2.8,3.0,3.2,3.2,3.4$, and $3.6 \mathrm{~cm}\left(1.8^{\circ}\right.$, $1.9^{\circ}, 2.0^{\circ}, 2.0^{\circ}, 2.2^{\circ}$, and $2.3^{\circ}$ ). The procedure for these practice trials conformed to the following experimental trials except that no contextual elements were presented. For the simultaneous condition, the complete stimulus with contextual wings and focal length drawn together (or a practice or filler stimulus line) was placed in one field, a comparison series was placed in another field, and the two fields were simultaneously illuminated for $5 \mathrm{sec}$. For the delay conditions, the contextual stimulus and/or fixation point was presented in one field for $5 \mathrm{sec}$. This display was then terminated and followed either immediately or at a variable dark delay by presentation of a focal or filler (or practice) stimulus line simultaneously with the comparison series. The focal or filler (or practice) stimulus was placed in a second field, the comparison series was placed in a third field, and the two fields were simultaneously illuminated for $5 \mathrm{sec}$.

The subjects selected a match from a series of 11 comparison lengths presented simultaneously with the standard length (practice, filler, or focal stimulus line). The comparison series ranged from 2.7 to $3.7 \mathrm{~cm}$ in $1-\mathrm{mm}$ increments $\left(1.7^{\circ}-2.4^{\circ}\right.$ in $.06^{\circ}$ increments). Judgments were reported by verbalization of the letter associated with the matching comparison. The subjects were told that they could report two letters if they felt that the matching extent fell between two comparison lines, but only rarely availed themselves of this option. As shown in Figure 3, context, standard and fixation points were centered in the left half of the available visual field, which measured $12.1 \times 15.2 \mathrm{~cm}\left(7.7^{\circ} \times 9.6^{\circ}\right)$ and the comparison series was left justified, $6.0 \mathrm{~cm}\left(3.8^{\circ}\right)$ from the center of the left half of the field, with a $.5-\mathrm{cm}\left(.3^{\circ}\right)$ vertical separation between lines of the series. The (dark) intertrial interval was $10 \mathrm{sec}$ for the simultaneous condition and $20 \mathrm{sec}$ for the delay conditions. Also, the subjects in the delay conditions were given a 2-min break between Experimental Blocks 2 and 3, during which time they were told to lean back in their chair and "rest your eyes."

\section{Results and Discussion}

Judgments of the 3.2-cm "no-context" practice stimuli were averaged to provide an estimate of the point of subjective equality (PSE) for each subject. The mean of these estimates was the same $(3.1 \mathrm{~cm})$ for both experiments, and an analysis of variance (ANOVA) indicated no differences in this baseline among groups for either Experiment $1[F(3,76)=1.38]$ or Experiment $2[F(3,76)$ $=.98]$. Thus, the subjects in the present experiments showed about the same underestimation of objective focal length that was found by Jordan and Uhlarik (1986, Experiment 1). To control for this underestimation, the data were expressed as deviations from each subject's PSE and these measures of illusion were subjected to a threeway ANOVA (group $\times$ contextual length $\times$ trial block), separately for each experiment.

Experiment 1. The only significant sources of variance in the first experiment were the main effects for group $[F(3,76)=2.79, p<.05]$, contextual length $[F(3,228)=$ $14.14, p<.001]$, and trial block $[F(3,228)=5.75, p<$ $.005]$, and the interaction of group $\times$ contextual length $[F(9,228)=29.66, p<.001]$. Because the effect of contextual length did not vary significantly over trial blocks $[F(9,684)=.63]$ for the four groups $[F(27,684)=1.29]$, the data are shown averaged over trial blocks in Figure 4. As can be seen in Figure 4, the group $\times$ contextual length interaction consisted largely of opposite effects of contextual length for the simultaneous and delay groups. Increasing contextual length produced increases in focal judgments for the simultaneous group (i.e., assimilation) 
and decreases in focal judgments for the delay groups (i.e., contrast).

A secondary analysis of only the data from the delay groups supports the conclusion that, though small, the effect of contextual length for these groups was reliable. The main effect of contextual length was the only significant source of variance in this analysis $[F(3,171)=18.00$, $p<.001]$. The effect of contextual length was not significantly different for the three groups $[F(6,171)=.83]$ and the overall differences among delay groups (i.e., main effect) appearing in Figure 4 were not very reliable. Thus, minimal temporal separation (i.e., 0-sec delay) of contextual and focal elements was sufficient to produce contrast, and longer delays up to $1 \mathrm{sec}$ did not significantly increase the magnitude of contrast effects.

Experiment 2. The only significant sources of variance in the second experiment were main effects for contextual length $[F(3,228)=25.17, p<.001]$ and trial block $[F(3,228)=3.32, p<.05]$, with interactions of group $\times$ contextual length $[F(9,228)=38.00, p<.001]$ and group $\times$ trial block $[F(9,228)=2.52, p<.01]$. Because the effect of contextual length did not vary significantly over trial blocks $[F(9,684)=1.74]$ for the four groups $[F(27,684)=1.05]$, the data are shown averaged over trial blocks in Figure 5. As in Experiment 1, with increasing contextual length, focal length judgments increased for the simultaneous condition (i.e., assimilation), but decreased for delay conditions (i.e., contrast). A secondary analysis of the delay groups' data revealed only a main effect for contextual length $[F(3,171)=13.77, p<$ $.001]$, with no differential effects of contextual length among the three groups $[F(6,171)=1.21]$ and no overall difference among groups $[F(2,57)<1.00]$. Thus, temporal delays up to $2 \mathrm{sec}$ did not increase the magnitude

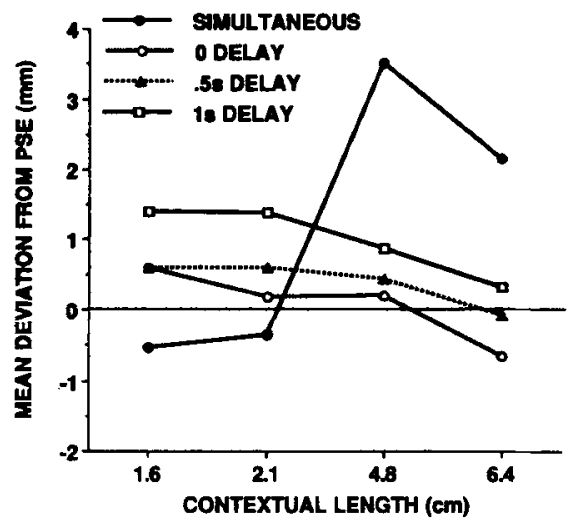

Figure 4. Mean deviation from point of subjective equality (PSE) for judgments of focal length (central axis) as a function of inward wings produced by $1.6-$ and $2.1-\mathrm{cm}$ contextual lengths $\left(1.0^{\circ}\right.$ and $\left.1.3^{\circ}\right)$ and outward wings produced by 4.8 - and $6.4-\mathrm{cm}$ contextual lengths $\left(3.1^{\circ}\right.$ and $4.1^{\circ}$ ) for conditions in which contextual elements preceded the focal line by a dark interval (.5- or 1-sec delay), the focal line immediately followed contextual elements ( 0 -sec delay), or contextual and focal elements appeared together (simultaneous). Data are from Experiment 1.

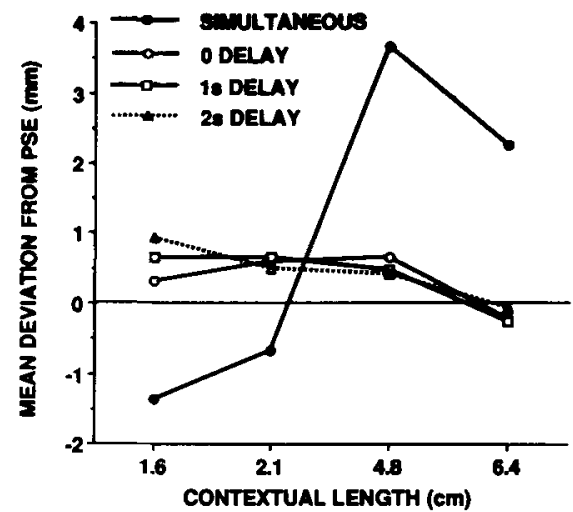

Figure 5. Mean deviation from point of subjective equality (PSE) for judgments of focal length (central axis) as a function of inward wings produced by $1.6-$ and $2.1-\mathrm{cm}$ contextual lengths $\left(1.0^{\circ}\right.$ and $\left.1.3^{\circ}\right)$ and outward wings produced by 4.8 - and $6.4-\mathrm{cm}$ contextual lengths $\left(3.1^{\circ}\right.$ and $\left.4.1^{\circ}\right)$ for conditions in which contextual elements preceded the focal line by a dark interval (1- or 2-sec delay), the focal line immediately followed contextual elements (0-sec delay), or contextual and focal elements appeared together (simultaneous). Data are from Experiment 2.

of contrast effects beyond that obtained with a 0 -sec delay between contextual and focal elements.

The significant main effect of trial blocks and group $\times$ trial block interaction could mean that, with task repetition, illusion magnitude changed, or that the subjects' bias in estimation of objective focal length changed, perhaps differently for the different groups. An examination of responses to the 2.8- and 3.6-cm no-context practice and filler stimuli supports the second interpretation. These stimuli were judged as being different in size $[F(1,76)=$ $1,528.83, p<.001]$, and judgments increased over trials $[F(4,304)=12.55, p<.001]$ differently for the two stimuli $[F(4,304)=14.77, p<.001]$. Mean responses for practice trials and the four experimental trials were $2.9,2.8,2.8,2.8$, and 2.9 for the $2.8-\mathrm{cm}$ focal length and $3.3,3.4,3.5,3.5$, and 3.5 for the $3.6-\mathrm{cm}$ focal length. Thus, underestimation of objective focal length occurred only for the longer stimulus and tended to disappear in later trials. It seems reasonable that a similar decrease in underestimation of objective focal length may have $\alpha$ curred for the 3.2-cm target length. If such undetected shifts in baseline occurred, all of the curves displayed in Figure 5 would be shifted upward; that is, the zero reference value would be too low relative to the curves. Also, the nonsignificant differences among delay conditions that appeared in Experiment 1 (see Figure 4) may have been due to individual differences among the groups in baseline shift.

The possibility of undetected baseline shifts does not change the general conclusion that the effect of increasing contextual length is in opposite directions for the simultaneous and delay conditions or that increases in delay up to $2 \mathrm{sec}$ have no appreciable effect. It does mean, however, that the relative magnitude of these assimila- 
tion and contrast effects for smaller (wings-inward) and larger (wings-outward) contexts may be misrepresented in Jordan and Uhlarik's (1986) data (see Figure 1) as well as in the present data. The baseline measurement procedure was changed in Experiment 3 to test for such baseline shifts and provide a better estimate of the relative magnitude of contrast effects.

The data from Experiments 1 and 2 demonstrate that even minimal delay $(0 \mathrm{sec})$ between contextual and focal elements was sufficient to reverse the usual assimilation illusion and these contrast effects were not enhanced by delays up to $2 \mathrm{sec}$. These results suggest that the processes responsible for contrast effects operate at a level of representation that is higher than that involved in processes responsible for assimilation effects. Assimilation may reflect the natural averaging of stimulus attributes involved in the organizational processes (constrained computations) that produce unitized object-level representations. Contrast may reflect the natural enhancement of differences among object representations involved in computing spatial relationships among discrete objects in the visual field. In this view, differencing operations should be activated and contrast effects should occur whenever the content of focal attention changes (e.g., with an eye movement), provided that other conditions are present (e.g., there is sufficient time) to enable organizational processing to be completed within each focus of attention. The 5-sec duration of contextual and focal elements was surely sufficient for perceptual organization (with consequent assimilation effects) and the instantaneous change in the 0-sec delay condition was sufficient to mark unique representations of contextual and focal elements (with consequent maximal contrast effects).

\section{EXPERIMENT 3}

The results of the first two experiments suggest that the representational basis for contrast effects is high-level and long-lived. In Experiment 3, we attempted to determine the time required to develop such high-level object representations by manipulating the presentation time of preceding contextual elements. Processing was restricted to contextual presentation time by a pattern mask in the interval before the following focal element. The contextual presentation time at which contrast effects first appear should provide an estimate of the temporal duration for development of the representational basis of contrast effects. Because Jordan and Uhlarik (1986) found no differences in contrast effects for contextual stimulus durations of 5 and $60 \mathrm{sec}$, shorter durations were investigated in the present experiment.

\section{Method}

Conditions for the third experiment are illustrated in Figure 6. This experiment was similar to the first two experiments with the following exceptions. Only a single .1-sec delay condition was tested and duration of the contextual array was the principal manipulation. Four random groups (20 subjects each) experienced contextual elements for $.1, .5,1$, or $5 \mathrm{sec}$, followed immediately by a .1 -sec duration presentation of a pattern mask, and then the focal element was presented simultaneously with the comparison series. The pattern mask consisted of a horizontally oriented rectangle measuring $6.5 \times 2.0 \mathrm{~cm}\left(4.1^{\circ} \times 1.3^{\circ}\right)$, which enclosed three " $\mathrm{X}$ " shapes formed by $4-\mathrm{cm}$ lines crossing to form $60^{\circ}$ horizontal angles. The two outside Xs were positioned such that they would overlay the contextual stimuli if presented simultaneously. To control for changes in baseline over the course of an experimental session, experimental trials included an instance of the $3.2-\mathrm{cm}$ focal length without context, in addition to the four instances of this focal length combined with preceding context and the two no-context filler lengths. To equate the number of practice and experimental stimuli, a third repetition of the $3.2-\mathrm{cm}$ practice stimulus was added. Finally, the dark intertrial interval was shortened to $15 \mathrm{sec}$.

\section{Results and Discussion}

An examination of responses to the $3.2-\mathrm{cm}$ no-context control stimulus confirmed the suspicion of baseline changes over blocks of trials $[F(4,304)=9.03, p<.001]$. Mean judgments were $3.0,3.0,3.1,3.1$, and $3.2 \mathrm{~cm}$ for practice and Experimental Blocks 1-4, respectively. This trend
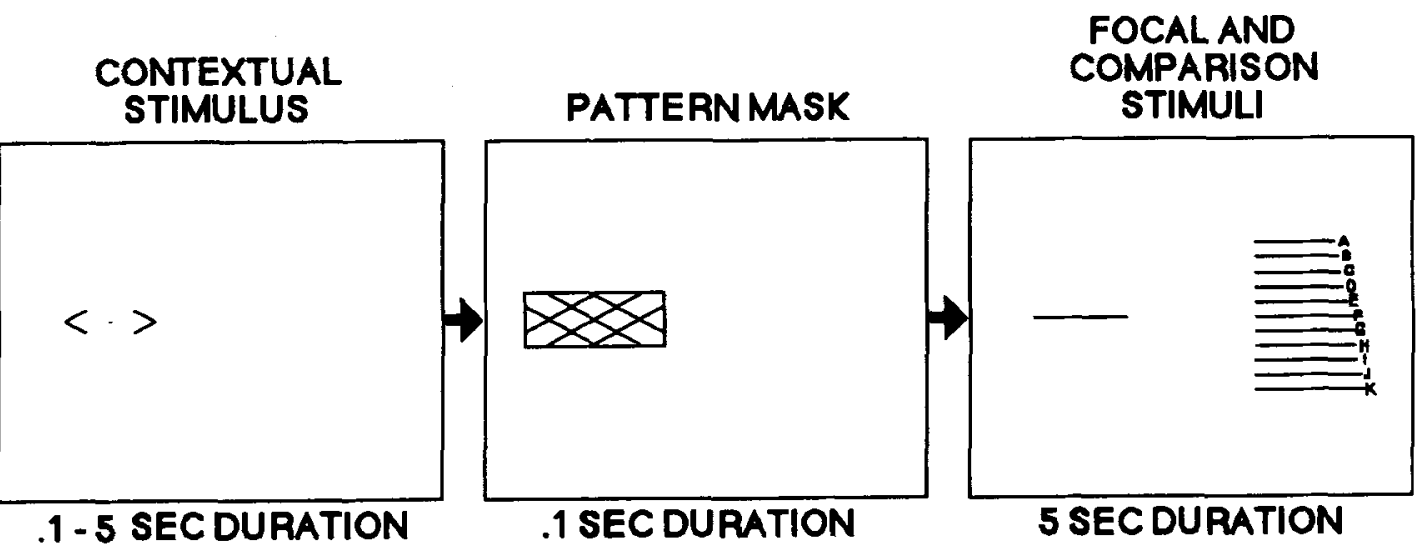

Figure 6. Illustration of experimental conditions for Experiment 3. In different conditions, the contextual wings were presented for a variable duration, followed immediately by a .1-sec pattern mask and then by a 5-sec presentation of the focal central axis together with the comparison series. Contextual lengths were the same as in Experiments 1 and 2 (see Figure 3). 
was not different for the four groups $[F(12,304<1.00]$ and there was no main effects for groups $[F(3,76)<1.00]$. To control for such bias for each subject, the data in each block of experimental trials were expressed as deviations from the control PSE measure in each block and these measures of illusion were subjected to a three-way ANOVA (group $\times$ contextual length $\times$ trial block).

The only significant sources of variance in this analysis were the main effect of context $[F(3,228)=7.34, p<$ $.001]$ and the group $\times$ context interaction $[F(9,228)=$ $2.70, p=.005]$. Because there was no effect of trial blocks $[F(3,228)=1.22]$ for any of the groups $[F(9,228)=.82]$ and the differential effects of context for groups did not change over trial blocks $[F(27,684)=.71]$, the data are shown averaged over trial blocks in Figure 7. As can be seen, the only consistent decrease in judged focal length with increasing context length (i.e., contrast) occurred for the 5-sec contextual duration. Subsequent analysis of only the data from the three shorter contextual durations revealed no significant effects, and analysis of the data for the 5 -sec contextual duration revealed only a significant effect of contextual length $[F(3,57)=10.39, p<.001]$. These results suggest that contextual durations greater than $1 \mathrm{sec}$ but perhaps less than $5 \mathrm{sec}$ are necessary to produce contrast effects.

Pollack (1964) found contrast effects with .5-sec presentations of contextual and focal elements with an interpolated dark delay of $.5 \mathrm{sec}$ and an unspecified but presumably long intertrial interval. ${ }^{3}$ Assuming that representational development continues after stimulus termination in the absence of a mask, the minimal processing time in Pollack's study for the contextual stimulus was $1 \mathrm{sec}$ and probably longer for the focal stimulus. Differences in lightness contrast levels and in measurement procedures may account

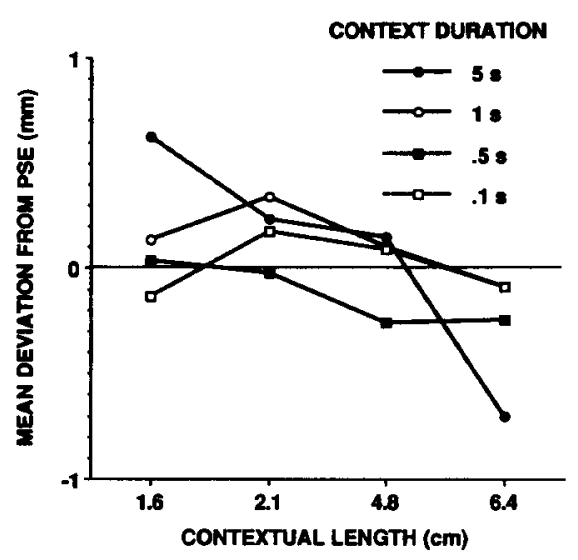

Figure 7. Mean deviation from point of subjective equality (PSE) for judgments of focal length (central axis) as a function of inward wings produced by $1.6-$ and $2.1-\mathrm{cm}$ contextual lengths $\left(1.0^{\circ}\right.$ and $\left.1.3^{\circ}\right)$ and outward wings produced by 4.8 - and $6.4-\mathrm{cm}$ contextual lengths $\left(3.1^{\circ}\right.$ and $\left.4.1^{\circ}\right)$ for conditions in which contextual wings were presented for durations of .1, .5, 1, or $5 \mathrm{sec}$, followed by a .1-sec pattern mask presentation before the focal length was presented for 5 sec. Data are from Experiment 3. for the absence of contrast effects with 1-sec contextual stimuli in the present study, but Pollack's data clearly suggest that the minimal duration for contrast effects is closer to $1 \mathrm{sec}$ than $5 \mathrm{sec}$.

The observation that contrast effects tend to be about the same magnitude for outward-wings and inward-wings configurations when change in baseline over trial blocks is controlled seems to contradict the prediction of larger contrast effects when assimilation effects are small. However, a baseline change in the observed direction (i.e., decreasing underestimation) would also change the observed magnitude of assimilation effects, decreasing assimilation for outward wings and increasing assimilation for inward wings. Thus, the asymmetry of contrast and assimilation illusions for inward and outward configurations observed in the first two experiments (see Figures 4 and 5) may have been artifactual (see also Adam \& Bateman, 1980; Christie, 1975). At present, the data are not sufficient to disconfirm the hypothesis.

\section{GENERAL DISCUSSION}

Presentation time for the preceding contextual wings on the order of $1 \mathrm{sec}$ or more was needed to produce contrast effects in judgments of the following focal axis (Experiment 3) and, given sufficient presentation time, these contrast effects were largely unaffected by the length of the temporal delay between contextual and focal elements, appearing equally strong for delays between 0 and $2 \sec (\mathrm{Ex}-$ periments 1 and 2). These results are consistent with a representational basis for contrast effects that is high-level and long-lived.

The Müller-Lyer contrast illusion may reflect the inadvertent error arising from basing judgments about particular objects on information about attribute differences among objects. Similarly, the Müller-Lyer assimilation illusion may reflect the error inherent in basing judgments about parts of objects on information about the average attributes of objects. Such judgmental errors may be the natural consequence of constrained computations that make explicit information required for certain common tasks, but at the expense of obscuring information required for less common tasks. In such cases, the visual system does the best job it can with the available information, and "illusion" is the name we give the resultant "error."

Attribute averaging and differencing are not the only constrained computations that may underlie illusions. Assimilation and contrast effects may be assumed to reflect the error inherent in constrained computation when the resulting information is used in tasks for which it is ill-suited, but another class of illusory effects may arise when constraints are inappropriately applied; that is, when the stimulus situation actually violates the usual constraints. For example, implicit knowledge of projective geometry may normally be applied to constrain interpretation of retinal projections (cf. Malik, 1987; Waltz, 1975), but illusions may result when such constraints are applied to twodimensional stimuli (cf. Gregory, 1963; Gregory \& Harris, 
1975; see also Redding \& Hawley, 1992; Warren \& Bashford, 1977). Computational theory offers a broad framework within which the variety of visual illusions may be understood.

\section{REFERENCES}

ADAm, J., \& Bateman, L. (1980). Control stimuli in investigations of the acute-angled and obtuse-angled Müller-Lyer illusions. Perception, 9, 467-474.

BIEDERMAN, I. (1987). Recognition-by-components: A theory of human image understanding. Psychological Review, 94, 115-147.

Brigell, M., \& UHLARIK, J. (1979). The relational determination of length illusions and length aftereffects. Perception, 8, 187-197.

Chinng, C. (1968). A new theory to explain geometrical illusions produced by crossing lines. Perception \& Psychophysics, 3, 174-176.

Christie, P. (1975). Asymmetry in the Mueller-Lyer illusion: Artifact or genuine effect? Perception, 4, 453-457.

COREn, S. (1970). Lateral inhibition and geometric illusions. Quarterly Journal of Experimental Psychology, 22, 274-278.

COREN, S. (1986). An efferent component in the visual perception of direction and extent. Psychological Review, 93, 391-410.

COREN, S., \& Girgus, J. S. (1978). Seeing is deceiving: The psychology of visual illusions. Hillsdale, NJ: Erlbaum.

COREN, S. , Girgus, J. S. (1980). Principles of perceptual organization and spatial distortion: The Gestalt illusions. Joumal of Experimental Psychology: Human Perception \& Performance, 6, 404-412.

Ebert, P. C., \&ollack, R. H. (1972), Magnitude of the MuellerLyer illusion as a function of lightness contrast, viewing time, and fundus pigmentation. Psychonomic Science, 26, 347-348.

EBerT, P. C., \& Pollack, R. H. (1973). Some factors affecting magnitude of the Mueller-Lyer illusion. Perceptual \& Motor Skills, 37, 433-434.

Erlebacher, A., \& Sekular, R. (1974). Perceived length depends on exposure duration: Straight lines and Müller-Lyer stimuli. Journal of Experimental Psychology, 103, 724-728.

GrRGUS, J. S. , COREN, S. (1982). Assimilation and contrast illusions: Differences in plasticity. Perception \& Psychophysics, 32, 555-561.

GREGORY, R. L. (1963). Distortion of visual space as inappropriate constancy scaling. Nature, 199, 678-680.

Gregory, R. L., \& HaRRIs, J. P. (1975). Illusion-destruction by appropriate scaling. Perception, 4, 203-220.

HoLding, D. H. (1970). A line illusion with irrelevant depth cues. American Joumal of Psychology, 83, 280-282.

ItTELSON, W. H., \& KilPatrick, F. P. (1951). Experiments in perception. Scientific American, 185, 54-67.

JoRDAN, K., \& ENGUSH, P. W. (1989). Simultaneous sampling and length contrast. Perception \& Psychophysics, 46, 546-554.

JoRDAN, K., \& HALEBLIAN, J. (1988). Orientation specificity of length assimilation and contrast. Perception \& Psychophysics, 43, 446-456.

JORDAN, K. \& SCHIANO, D. J. (1986). Serial processing and the parallellines illusion: Length contrast through relative spatial separation of contours. Perception \& Psychophysics, 40, 384-390.

JORDAN, K., \& UHLARIK, J. (1985). Assimilation and contrast of perceived length depend on temporal factors. Perception \& Psychophysics, $37,447-454$.

JORDAN, K., \& UhLARIK, J. (1986). Length contrast in the Müller-Lyer figure: Functional equivalence of temporal and spatial separation. Perception \& Psychophysics, 39, 267-274.

MaLIK, J. (1987). Interpreting line drawings of curved objects. International Journal of Computer Vision, 1, 73-103.

MARR, D. (1982). Vision: A computational investigation into the human representation and processing of visual information. San Francisco: Freeman.

MARR, D., \& PoGgio, T. (1977). From understanding computation to understanding neural circuitry. Neurosciences Research \& Progress Bulletin, 15, 470-488.

MiNSKY, M., \& PAPERT, S. (1969). Perceptrons: An introduction to computational geometry. Cambridge, MA: MIT Press.
Morgan, M. I. \& Glennerster, A. (1991). Efficiency of locating centres of dot-clusters by human observers. Vision Research, 31, 2075-2083.

Morgan, M. J., Hole, G. J., \& Glennerster, A. (1990). Biases and sensitivities in geometrical illusions. Vision Research, 30, 1793-1810.

Piaget, J. (1969). The mechanisms of perception (G. N. Seagrine, Trans.). New York: Oxford University Press.

Pollack, R. H. (1964). Simultaneous and successive presentation of elements of the Mueller-Lyer figure and chronological age. Perceptual \& Motor Skills, 19, 303-310.

PosNer, M. I. (1980). Orienting of attention. Quarterly Journal of Experimental Psychology, 32, 3-25.

Pressey, A. W. (1970). The assimilation theory applied to a modification of the Müller-Lyer illusion. Perception \& Psychophysics, 8 , 411-412.

Pressey, A. W -(1972). The assimilation theory of geometric illusions An additional postulate. Perception \& Psychophysics, 11, 28-30.

Pressey, A. W. (1974). Evidence for the role of attentive field in the perception of illusions. Quarterly Joumal of Experimental Psychology, 26, 464-471.

Pressey, A. W., \& Di Lollo, V. (1978). Effects of distance between standard and comparison lines on the Müller-Lyer illusion. Perception \& Psychophysics, 24, 415-419.

Pressey, A., \& Martin, N. S. (1990). The effects of varying fins in Müller-Lyer and Holding illusions. Psychological Research, 52, 46-53.

Redding, G. M., \& Hawley, E. A. (1992, May). Length illusion in half-figure Müller-Lyer stimuli. Paper presented at the meeting of the Midwestern Psychological Association, Chicago, IL.

SCHULtz, T. (1991). A microgenetic study of the Müller-Lyer illusion. Perception, 20, 501-512.

Solso, R. L., Cantrell, J., \& Paolini, N. (1989, November). The future of an illusion and visual persistence. Paper presented at the meeting of the Psychonomic Society, Atlanta, GA.

Stuart, G. W., Day, R. H., \& Dickinson, R. G. (1984). Müller-Lyer: Illusion of size or position? Quarterly Journal of Experimental Psychology, 36A, 663-672.

Treisman, A. (1985). Preattentive processing in vision. Computer Vision, Graphics, \& Image Processing, 31, 156-177.

Treisman, A. M., \& Gelade, G. (1980). A feature-integration theory of attention. Cognitive Psychology, 12, 97-136.

Ullman, S. (1984). Visual routines. Cognition, 18, 97-159.

WALTZ, D. (1975). Understanding line drawings of scenes with shadows. In P.H. Winston (Ed.), The psychology of computer vision (pp. 1992). New York: McGraw-Hill.

WARREN, R. M., \& BASHFORD, J. A. (1977). Müller-Lyer illusions: Their origin in processes facilitating object recognition. Perception, 6, 615-626.

\section{NOTES}

1. Two other studies (Schultz, 1991; Solso, Cantrell, \& Paolini, 1989) have temporally separated contextual and focal elements of the MüllerLyer illusion. However, neither study obtained quantitative measures of perceived size and it is not possible to identify the direction of illusory effects. The study by Schultz is further obscured by the questionable application of signal detection analysis to categorical responses. For these reasons, these studies are not further discussed.

2 . It should be noted that there are other ways to account for differential asymmetry for the two illusions. For example, because the asymmetry takes the form of greater overestimation than underestimation effects, it may reflect a logarithmic representation of length in the visual system (Brigell \& Uhlarik, 1979; Jordan \& Uhlarik, 1986).

3. It may be noted that Pollack (1964) used only outward wings, $.5 \mathrm{~cm}$ in length, at a $90^{\circ}$ angle on a $2.2-\mathrm{cm}$ focal axis, producing a contextual length of approximately $2.8 \mathrm{~cm}$.

(Manuscript received October 13, 1992; revision accepted for publication April 5, 1993.) 